\title{
Biomedical research and clinical practice - your journal
}

\author{
Kazuhisa Nishizawa* \\ Department of Clinical Laboratory Science, Teikyo University, Japan
}

This issue marks the beginning of the second volume of Biomedical Research and Clinical Practice (BRCP). We had four issues for volume 1 , but volume 2 is planned to have six issues a year. It is not something we can take for granted to announce this transition as countless open access journals are born nowadays, but few enjoy over 45 submissions as we did in 2016. On behalf of the editorial board members, I would like to express my gratitude to the authors, whose intriguing findings as well as insights and boldness in discussions helped BRCP to gain momentum that we enjoy today. Although we could not accept all the submitted manuscripts, all submissions gave us confidence and helped to shape the direction of this journal.

In 2016, the editorial board of BRCP organized 56 sections of research areas, each section comprising of several excellent scientists. This was an important development, as explicit listing of the sections not only helps authors in choosing BRCP as a venue but also reflects our spirit to maintain a broad scope of this journal. Of note, some sections still need more members. Not only do we seek your contribution as an author, but we would also like to hear from you if you are interested in our editorial board.

It was broadness of scope that we prioritized in trying to give unique features to this journal. While it would be too early to judge whether $\mathrm{BRCP}$ has done well in this regard, it is interesting to see some papers have shown interdisciplinary features. Kamiyama et al. [1] was a study from an intensive care unit that showed that septic patients require special consideration on the basal metabolic rate, but that this change was unexpectedly smaller than previously considered. For another example, Dawson et al. [2] reported paradoxical prothrombin time changes observed during a dual treatment with warfarin and rifampicin, suggesting complex effects by rifampicin metabolism on warfarinsensitive and insensitive vitamin $\mathrm{K}$ reduction (reactivation) pathways. Needless to say, interpretation of a constellation of symptoms and findings in clinics nearly always requires multidisciplinary discussions. Patients cannot limit their cases to one discipline and this is where the value of multidisciplinary journals stands. Providing a useful forum to diverse authors any time is an important objective of BRCP.

We held a special issue on gut microbiota in 2016. After my authoring a concise summary of biochemical aspects of low-grade endotoxemia, we gratefully published a review article by Suzuki and coworker [3] on the effects of commensal bacteria on Th17 activity and autoimmune diseases including Behçet's disease. Ample evidence now shows close relationship of mucosal immunity, commensal bacteria,

Copyright: (2017 Nishizawa K. This is an open-access article distributed under the terms of the Creative Commons Attribution License, which permits unrestricted use, distribution, and reproduction in any medium, provided the original author and source are credited. regulatory lymphocytes and Th17 cells. Following this, my group published review articles on diverse modalities immunosuppression by mesenchymal stem cells and on negatively regulating factors acting on Th17 cells [4]. As this area will be productive in years to come, we suspect that further discussions will be covered by this volume of BRCP.

As a reviewer for other journals, I personally had a chance to feel researchers' interest in learning algorithms applied to gene expression profile dataset from diverse diseases to find genes useful for distinguishing patients into subtypes and from healthy subjects. Although putting such an approach to clinical practice still requires a leap forward, it would be one of our long-term objectives to develop a bridge connecting medicine, molecular cellular biology and technologies for big data analysis. On the other hand, in clinics, even minor topics can become important. Researches on any types of disease have many frontiers where quests for time- and cost-efficiency motivate studies on biosensors, disease markers, reevaluation of diagnostic criteria, and tailor-made protocols of treatment. So, any diseases can become a starting point to build such a bridge. We also thank a few authors for contributing reports on issues on public health and medical economics that helped BRCP to keep its scope wide.

The future looks bright for the activity to link biomedical research and clinical practice, as it gains re-recognition as a natural and beneficial dynamics in medical research. Our sincere hope is that BRCP continues and, through this volume two, helps ambitious steps of medical researchers toward unbounded directions of work.

\section{References}

1. Kamiyama J, Takazawa T, Yanagisawa A, Kanamoto M, Tobe M, et al. (2016) Comparison between resting energy expenditure measured by indirect calorimetry and metabolic rate estimate based on Harris-Benedict equation in septic patients. Biomed Res Clin Prac 1: 123.

2. Dawson J, Dedigama M, Elliot DJ, Sorich M, Mangoni AA, et al. (2016) Prolonged induction of warfarin metabolism and a paradoxical INR response in a mitral valve replacement patient receiving rifampicin for infective endocarditis. Biomed Res Clin Prac 1: 112.

3. Shimizu J, Suzuki N (2016) Enhanced Th17 responses with intestinal dysbiosis in human allergic, inflammatory, and autoimmune diseases. Biomed Res Clin Prac 1: 113

4. Seki R, Nishizawa K (2016) Factors regulating Th17 cells: a review. Biomed Res Clin Prac 1: 122.

Correspondence to: Kazuhisa Nishizawa, Department of Clinical Laboratory Science, Teikyo University, Japan, Tel: +81-3-3964-1211, ext: 46136.

Received: January 02, 2017; Accepted: January 06, 2017; Published: January 09, 2017 